\title{
Loss of PHD3 in myeloid cells dampens the inflammatory response and fibrosis after hind-limb ischemia
}

\author{
Angelika Beneke ${ }^{1}$, Annemarie Guentsch ${ }^{1}$, Annette Hillemann ${ }^{1}$, Anke Zieseniss ${ }^{1}$, Lija Swain ${ }^{1}$ and Dörthe M Katschinski ${ }^{\star 1}$
}

Macrophages are essential for the inflammatory response after an ischemic insult and thereby influence tissue recovery. For the oxygen sensing prolyl-4-hydroxylase domain enzyme (PHD) 2 a clear impact on the macrophage-mediated arteriogenic response after hind-limb ischemia has been demonstrated previously, which involves fine tuning a M2-like macrophage population. To analyze the role of PHD3 in macrophages, we performed hind-limb ischemia (ligation and excision of the femoral artery) in myeloidspecific PHD3 knockout mice (PHD3 ${ }^{-1-}$ ) and analyzed the inflammatory cell invasion, reperfusion recovery and fibrosis in the ischemic muscle post-surgery. In contrast to PHD2, reperfusion recovery and angiogenesis was unaltered in PHD ${ }^{-1-}$ compared to WT mice. Macrophages from PHD3 ${ }^{-1-}$ mice showed, however, a dampened inflammatory reaction in the affected skeletal muscle tissues compared to WT controls. This was associated with a decrease in fibrosis and an anti-inflammatory phenotype of the PHD3 ${ }^{-1-}$ macrophages, as well as decreased expression of Cyp2s1 and increased PGE2-secretion, which could be mimicked by $\mathrm{PHD3}^{-l-}$ bone marrow-derived macrophages in serum starvation.

Cell Death and Disease (2017) 8, e2976; doi:10.1038/cddis.2017.375; published online 10 August 2017

Non-sterile as well as sterile inflictions of tissues can induce an inflammatory response. ${ }^{1}$ For both the innate immune system is the first line of defense, which is recruited to combat a potential threat. In the case of sterile inflammation the innate immune system supports tissue reconstitution. This has been implicated during the course of ischemic diseases such as myocardial infarction, stroke or peripheral arterial disease $(P A D){ }^{2,3} P A D$ is a devastating disorder with high rates of morbidity and mortality. Symptoms of PAD range from claudicatio intermittens to the loss of the inflicted extremity. Distal to the vessel occlusion an ischemia-induced inflammatory infiltrate develops in which neutrophils and macrophages are the key members. ${ }^{4}$ Hypoxia is a prominent feature of the inflammatory microenvironment due to increased oxygen demand and decreased supply. Hypoxia can actively affect inflammatory processes through the oxygen-sensitive regulation of the hypoxia-inducible factor (HIF) signaling pathway in multiple immune cell subtypes that are either resident within the inflamed tissue or have migrated from the oxygenated blood to the hypoxic inflammatory milieu. ${ }^{5} \mathrm{HIF}$ comprises two subunits, that is, the oxygen dependently regulated $\mathrm{HIFa}$ subunit and the constitutively expressed HIF- $1 \beta$ subunit. ${ }^{6}$ At the molecular level, HIFa is regulated by three well described prolyl-4-hydroxylase domain enzymes (PHD) 1-3. ${ }^{7}$ Under consumption of molecular oxygen, HIFa is hydroxylated by the PHDs subsequently leading to $\mathrm{pVHL}$-dependent ubiquitination and proteasomal degradation. ${ }^{8}$ In hypoxia, PHD-mediated hydroxylation is impaired by the lack of the co-substrate oxygen, which results in the stabilization of HIFa, heterodimerization with its $\beta$-subunit and activation of HIF target genes. Via those target genes, HIF induces metabolic adaptation, neovascularization but also shapes the ischemia-induced inflammation. ${ }^{9}$

There is clear evidence for differential roles of the PHD isoforms in immune cell subpopulations in both resting and activated states. ${ }^{10}$ Most of these analyses were performed in infectious disease models neglecting sterile inflammation. Further investigation is required to particularly understand the role of PHDs in sterile inflammation especially in light of the fact that hydroxylase inhibitors are tested for their tissue protective potential in case of ischemia-related diseases. ${ }^{11}$ Experimental hind-limb ischemia in mice, which is induced by femoral artery ligation, is a well-accepted disease model for studying the consequences of ischemia-induced inflammation, fibrosis, neovascularization and tissue reconstitution. ${ }^{12}$ Ubiquitious non-cell type-specific deletion of the PHDs in genetically modified mice or local depletion via injection of shRNAs in the ischemic muscles targeting the PHDs stabilize $\mathrm{HIF}-1 a$, promote neovascularization and improve perfusion in murine models of hind-limb ischemia. ${ }^{13,14}$ Thus, inhibition of PHDs is a potentially effective option for advanced PAD. Most interestingly, Takeda et al. ${ }^{15}$ reported that deleting PHD2 cell type specifically in myeloid cells likewise increased neovascularization. The three PHD variants in mammalia differ regarding organ-specific expression, their inducibility in hypoxia and other non-HIF-related functions. ${ }^{16}$ Especially, PHD3 has been associated with cell survival decisions and altered macrophage function. ${ }^{17}$ A myeloid-specific knockout of PHD3 affects cell survival and apoptosis of neutrophils and macrophages. ${ }^{18}$ In the context of bacterial lung or intestinal

\footnotetext{
${ }^{1}$ Institute of Cardiovascular Physiology, University Medical Center, Göttingen, Germany

*Corresponding author: DM Katschinski, Institute of Cardiovascular Physiology, University Medical Center, University of Göttingen, Humboldtallee 23,37073 Göttingen, Germany. Tel: +49 55139 9778; Fax: +49 55139 5895. E-mail: doerthe.katschinski@med.uni-goettingen.de

Received 11.4.17; revised 22.6.17; accepted 04.7.17; Edited by S Lavandero
} 
infections, it has been demonstrated that a myeloid-specific PHD3 knockout results in hyperinflammation. ${ }^{19,20}$ The consequences for a non-sterile ischemic inflammation, however, are unknown. Therefore, we analyzed the consequences of a hind-limb ischemia in myeloid-specific PHD3 knockout mice.

\section{Results}

Reperfusion and angiogenesis after hind-limb ischemia are unchanged in $\mathrm{PHD}^{-1-}$ mice. Surgical ligation and excision of the femoral artery in mice induces severe ischemia distally to the occlusion in the GM of the calf including intense inflammatory processes. The inflammation is accompanied by ischemia-induced angiogenesis and muscle tissue remodeling. Arteriogenesis, which is induced by altered shear-stress is precluded by the hind-limb ischemia model applied in our study as the pre-existing caudal femoral artery (A. profunda femoris) was excised. Activated macrophages contribute angiogenic and angiostatic factors in the process of ischemia-induced angiogenesis and thus perfusion recovery. The overall impact of angiogenesis and thus hind-limb perfusion can be assessed by LDPI of the feet, which also allows sequential analysis of the reperfusion capacity in one mouse. Accordingly, we performed LDPI with WT and $\mathrm{PHD}^{-1-}$ mice up to 28 days after hind-limb ischemia surgery (Figures $1 a$ and $b$ ). In both genotypes, perfusion was significantly impaired below $10 \%$ compared to the non-ligated leg in response to femoral artery ligation and recovered to around $70 \%$ on day 28 . Neither the time course nor the extent of reperfusion recovery was significantly different between WT and $\mathrm{PHD}^{-1-}$ mice. The unaltered angiogenic response in the $\mathrm{PHD}^{-/-}$mice was additionally verified by immunohistochemistry analysis of capillaries by Isolectin IB4 tracing of endothelial cells and costainings with Hoechst (nuclei stain) and anti-vinculin antibodies in the ischemic GM muscles on day 3 and 28 after hind-limb ischemia (Figures 1c and d). The number and the size of the capillaries were significantly increased on both days in the ligated compared to the unligated leg demonstrating that the induced ischemia indeed triggered an angiogenesis response, which however was unaltered comparing WT and $\mathrm{PHD}^{-1-}$ mice. This was likewise reflected in the analysis of necrotic toe development in consequence of the ischemic tissue destruction. Mice from both genotypes developed necrotic toes with no difference in the extent or number of necrotic toes per paw (Figure 1e).

Macrophage infiltration in the ischemic muscle is altered in PHD3 ${ }^{-I-}$ mice. Immediately after an ischemic injury, the innate immune system is activated and is responsible for recruitment of inflammatory cells from the circulation. We quantified the inflammatory infiltrate in the GM in the ischemic versus non-ischemic legs of WT and $\mathrm{PHD}^{-/-}$mice 1-7 days after hind-limb ischemia by CD11b staining and FACS analysis (Figure 2a). The leukocyte infiltrate was significantly increased 1 day after hind-limb ischemia, peaked at day 4 and was almost completely resolved on day 7 with no kinetic difference in WT and PHD3 ${ }^{-1-}$ mice. The number of CD11bpositive cells, however, was significantly lower in the
$\mathrm{PHD}^{-1-}$ mice. CD11b is an integrin family member and is expressed on the surface of many leukocytes including monocytes, neutrophils, natural killer cells, granulocytes, and macrophages. A sequential influx of neutrophils and macrophages upon injury is a hallmark of acute tissue damage. Neutrophils are the first line of defense; macrophages are subsequently needed to remove the invading neutrophils that undergo NETosis or apoptosis. Characterization of the infiltrating leukocytes by Ly-6G and F4/80 - CD11b costaining confirmed the sequential activation of neutrophils and macrophages peaking at day 1 and day 4, respectively (Figures $2 \mathrm{~b}$ and $\mathrm{c}$ ). Although the infiltration with neutrophils was unaltered in WT compared to $\mathrm{PHD}^{-1-}$ mice, the number of macrophages present in the ischemic muscle was significantly lower in the $\mathrm{PHD}^{-/-}$mice, demonstrating that mainly macrophages are responsible for the difference in the CD11b-positive inflammatory infiltrate.

Infiltrating macrophages have a major role in cellular and molecular events of fibrosis. Scarring is necessary to address the loss of tissues that cannot rapidly or only incompletely regenerate. A persistent accumulation of macrophages can prolong tissue inflammation and aggravate fibrosis, which is associated with a possible long-term loss of tissue function. The extent of fibrosis in the ischemic muscle after hind-limb ischemia was therefore analyzed (Figures $3 a$ and $b$ ). In line with the lower numbers of infiltrating macrophages, we observed significantly less fibrosis in the ischemic GM of the $\mathrm{PHD}^{-1-}$ compared to WT mice.

PHD3 $^{-1-}$ macrophages in the ischemic muscle have an anti-inflammatory phenotype. Changing tissue environments, which occur after the onset of tissue injury, shape the phenotype of macrophages to provide them with additional functional properties like altered life span or proand anti-inflammatory characteristics. This helps to meet the tissues need in addressing the damage. As a defining hallmark of macrophages, this plasticity allows them to adjust their effector functions. As PHD3 has been described to alter viability of myeloid cells, we analyzed markers for apoptosis and necrosis in the macrophages isolated from the inflamed muscles after hind-limb ischemia. We found a significant proportion of macrophages with markers characteristic for late apoptosis, however, without any difference when comparing WT and $\mathrm{PHD}^{-/}$macrophages (Figure 4a). In a previous report, we have demonstrated that a knockout of PHD2 in macrophages results in a HIF-mediated metabolic phenotype associated with impaired migration and phagocytosis, which might result in a delayed inflammatory infiltrate. ${ }^{21}$ A similar migratory impairment of the $\mathrm{PHD}^{-/-}$macrophages was excluded in single-cell migration experiments (Figure 4b).

The existence of a plethora of macrophage polarization states is critical for the adequate onset, regulation, and resolution of inflammatory responses. ${ }^{22} \mathrm{M} 1$ - and $\mathrm{M} 2$-polarized macrophages are the extremes of a continuum of intermediate cells, which can be seen after stimulation of macrophages with certain cytokines in vitro. In vivo macrophages are more or less skewed into the direction of one of these extremes. We analyzed the presence of CD68 and CD206 by FACS analysis as characteristic markers for M1- and M2-like polarized cells, 

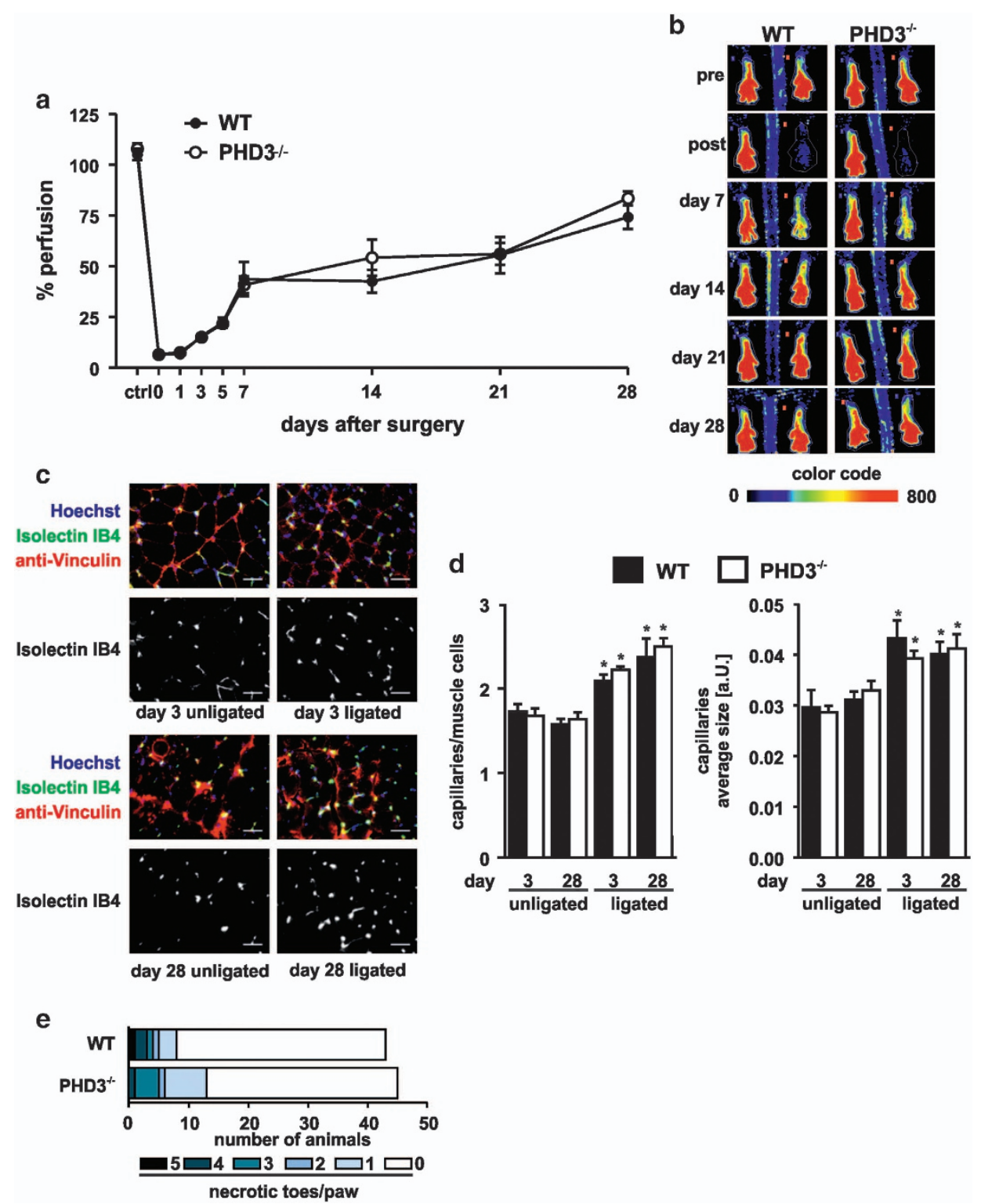

Figure 1 Perfusion recovery and angiogenesis after hind-limb ischemia are unchanged in $\mathrm{PHD}^{-1-}$ mice. (a) Perfusion was measured from Laser Doppler images before, directly after and 1, 3, 5, 7,21 and 28 days after hind-limb ischemia surgery. Groups included at least seven mice for each time point and each genotype. (b) Exemplary images of Laser Doppler measurements are shown; color code represents the intensity of perfusion (arbitrary intensity units). (c) Cryosections of the gastrocnemius muscle isolated from the ischemic (ligated) and non-ischemic control (unligated) leg were stained for isolectin IB4, vinculin and nuclei (Hoechst) to visualize capillaries and muscle cells. Scale bars represent $50 \mu \mathrm{m}$. (d) The amount and average area of capillaries of sections of at least five mice per group were quantified using ImageJ. (e) The number of necrotic toes on the ligated legs was quantified after hind-limb ischemia surgery. Graphs show mean \pm S.E.M. with ${ }^{\star} P<0.05$

respectively, on CD11b/F4/80-positive cells in the ischemic GM (Figures $4 \mathrm{c}$ and d). Similar to the kinetic of the macrophage infiltrate, CD206 expression peaked on day 4 with an inverse pattern in expression of CD68. In line with the dampened inflammatory infiltrate in the $\mathrm{PHD}^{-/-}$mice after hind-limb ischemia, $\mathrm{PHD}^{-/-}$macrophages displayed significantly higher expression of CD206 on day 4 and 5 compared to WT macrophages supporting an antiinflammatory phenotype.

Differential expression of Cyp2s1 in $\mathrm{PHD}^{-1-}$ macrophages. Understanding of the macrophage population switch is an attractive target to manipulate inflammation and to reduce fibrosis and scarring, which would improve healing and long-term tissue function. To gain insight into the differential inflammatory response of WT and $\mathrm{PHD}^{-1-}$ macrophages, we FACS-sorted CD11b-F4/80-positive cells from the ischemic GM of 3 WT and $5 \mathrm{PHD}^{-1-}$ mice on day 5 after hind-limb ischemia, isolated RNA and performed RNAseq analysis. The bioinformatic analysis revealed in total 10 differentially expressed RNAs (Figure 5a). The RNA-seq protocol included an mRNA fragmentation approach prior to sequencing to gain sequence coverage of the whole transcript. The total number of reads for a given transcript is thus proportional to the expression level of the transcript multiplied by the length of the transcript, which is reflected in 

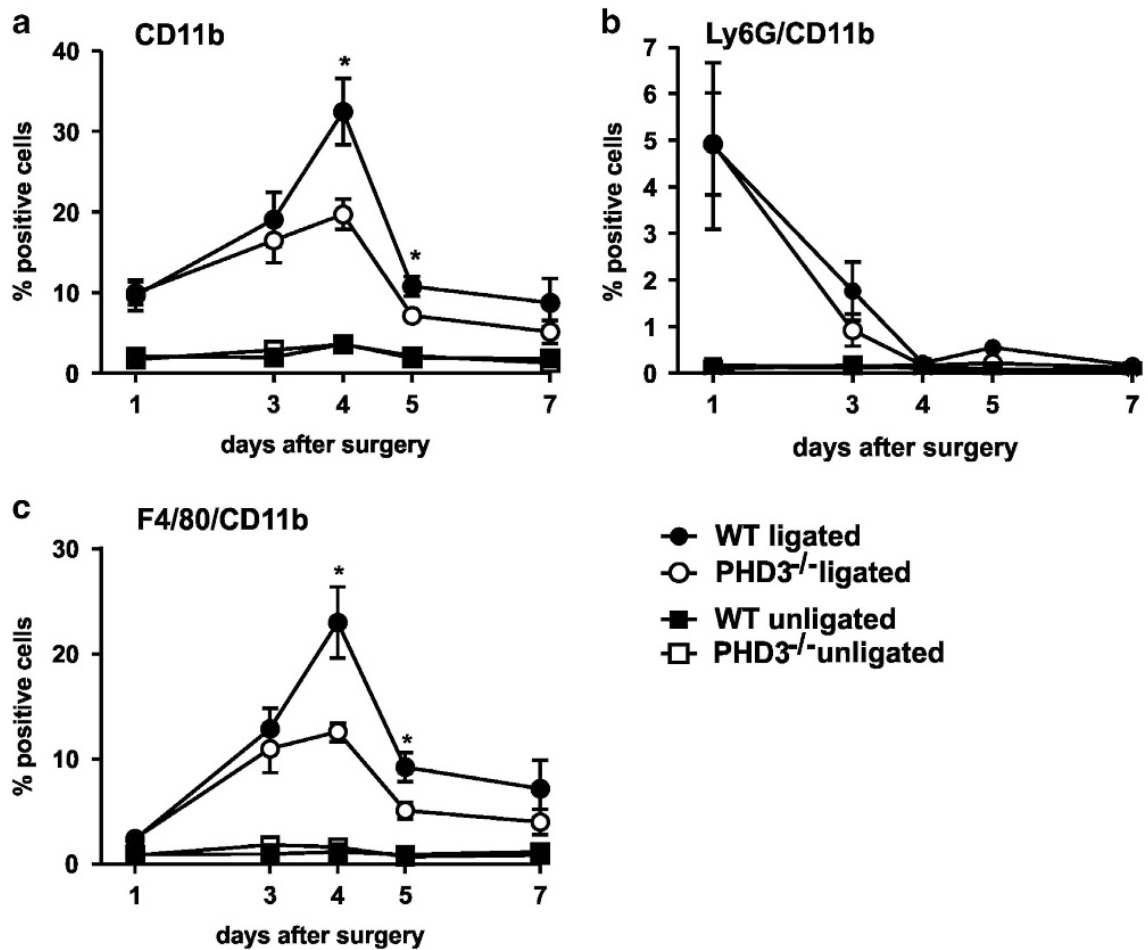

Figure $2 \mathrm{PHD}^{-1}$ - mice show a dampened leukocyte infiltration into the ischemic muscle after hind-limb ischemia. Leukocyte infiltration was analyzed via flow cytometry over the course of 7 days after surgery within the gastrocnemius muscle of the ischemic (ligated) and non-ischemic control (unligated) legs of wild type (WT) and PHD $3^{-1-}$ mice. Leukocytes were identified as CD11b-positive cells (a), whereas neutrophils were identified as Ly-6G CD11b-positive cells (b) and macrophages as F4/80 CD11b-positive cells (c). At least five mice were analyzed per group. Graphs represent mean \pm S.E.M. with ${ }^{\star} P<0.05$

a

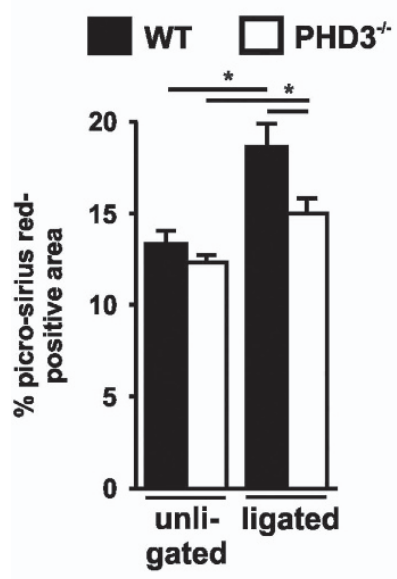

b

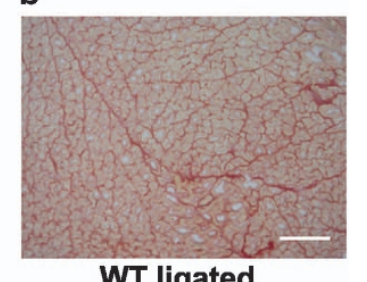

WT ligated

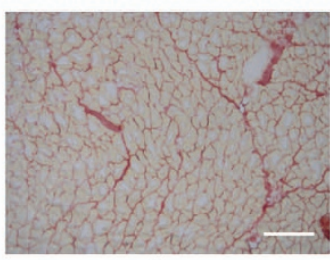

PHD3 $^{+}$ligated

Figure $3 \mathrm{PHD}^{-1-}$ mice show less fibrosis after hind-limb ischemia. Fibrosis was visualized using picro-sirius red staining on cryosections of gastrocnemius muscles 28 days after surgery (a). The picro-sirius red positive area was quantified via ImageJ (b). seven mice per group were analyzed. Scale bars represent $200 \mu \mathrm{m}$. Graphs show mean \pm S.E.M. with ${ }^{*} P<0.05$

the reads per kilobase transcript per million (RPKM). Of note, the cut-off chosen (base mean $>50$ ) for analysis did not allow PHD3 to appear in the list of differentially expressed candidates based on its low overall expression level reflected by a base mean of 18 and RPKM value below 1 . Among the identified candidates miRNA511 and Cyp2s1 were previously

described to be associated with inflammatory functions. Although the differential expression of miRNA511 could not be verified in in vitro differentiated BMDM cultured in normoxia and in simulated ischemic conditions, that is, serum starvation (data not shown), the differential expression of Cyp2s1 was also seen in the cultured macrophages (Figure $5 \mathrm{~b}$ ). Interestingly, the difference was only detectable in serum starvation demonstrating that the PHD3-mediated differential expression is only applicable to those conditions. Cyp2s1 is a member of the cytochrome $P$ (Cyp)450 superfamily of enzymes. Cyp2s1 is highly expressed in epithelial tissues of the respiratory, gastrointestinal, urinary tracts, and skin as well as in leukocytes of the monocyte/macrophage and lymphocyte series. ${ }^{23}$ Extra hepatic CYP epoxygenases can utilize endogenous substrates such as arachidonic acid, linoleic acid, eicosapentaenoic acid and docosahexenoic acid to generate bioactive lipid mediators. ${ }^{24}$ In this context, Cyp2s1 is an important enzyme in the metabolism of COXderived prostaglandins including PGE2 at nanomolar concentrations, and thus has an important role in modulating inflammatory processes including macrophage phagocytosis. We verified that PGE2 expression and phagocytosis were indeed affected in the $\mathrm{PHD}^{-1-}$ macrophages (Figures $5 \mathrm{c}$ and d). We found elevated Cyp2s1 expression in serum starvation. This response was significantly blunted in the $\mathrm{PHD3}^{-1-}$ BMDM. In line with the increased expression of Cyp2s1 in serum-starved WT BMDM, PGE2 levels were decreased in this condition. In contrast, the PGE2 levels in the $\mathrm{PHD}^{-/-}$BMDM were significantly higher in line with the 


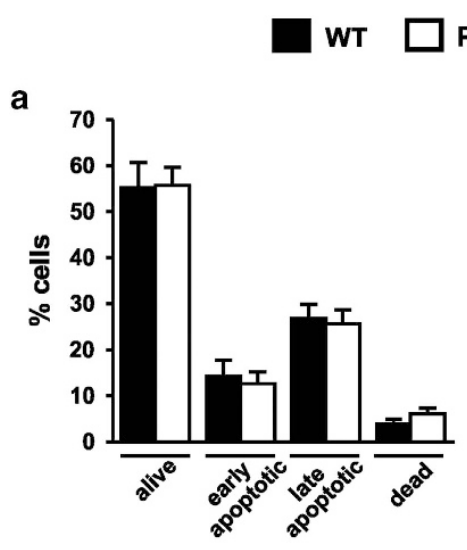

PHD3 ${ }^{-1}$
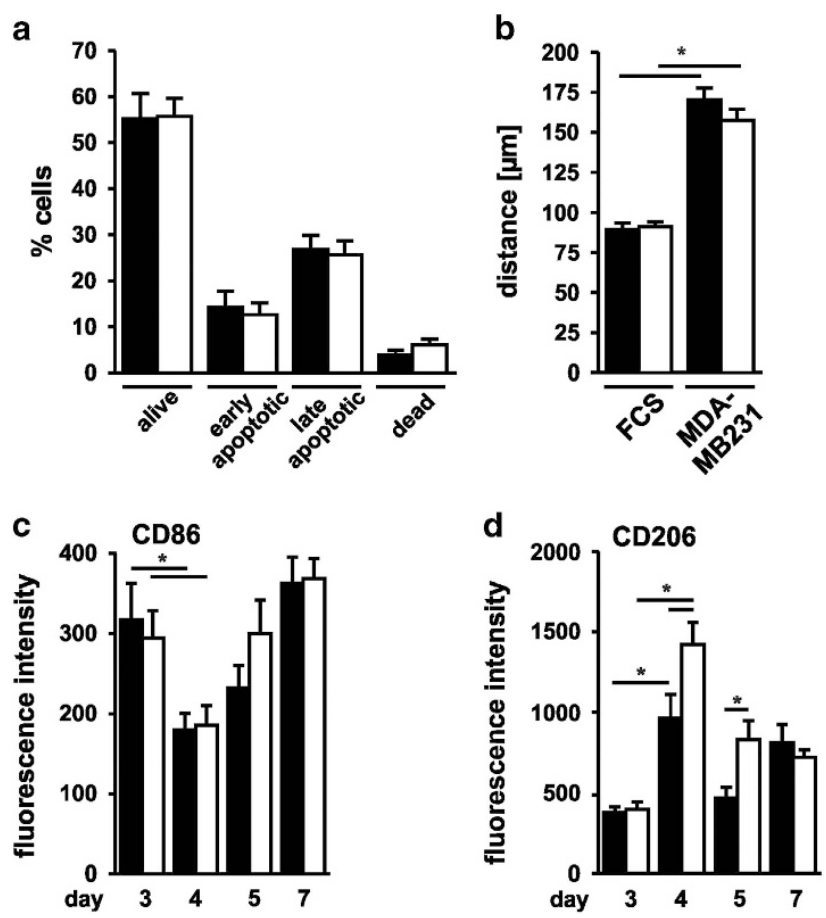

Figure $4 \mathrm{PHD}^{-1-}$ macrophages are more M2-polarized after hind-limb ischemia surgery compared to wild type (WT) and show unaltered apoptosis and migratory behavior. (a) Apoptosis was analyzed in F4/80 CD11b-positive macrophages isolated from the gastrocnemius muscles (GM) of WT and $\mathrm{PHD}^{-1-}$ mice 4 days after hind-limb ischemia via stainings with Zombie green and Annexin V. Dead cells were identified as Annexin V - Zombie green high, late apoptotic cells as Annexin V+ Zombie green high, early apoptotic cells as Annexin V+ Zombie green low and living cells as Annexin V - Zombie green low cells. At least six animals were included per genotype. (b) The migratory capacity of BMDM was analyzed in a singlecell migration assay via the accumulated distance cells traveled within $6 \mathrm{~h}$. The graph is representative for at least three independent experiments. F4/80 CD11b-positive macrophages isolated from the GM after surgery were analyzed for their geometric mean fluorescence intensity of CD86 (c, M1-marker) and CD206 (d, M2-marker). At least five animals were included per genotype. Graphs show mean \pm S.E.M. with ${ }^{*} P<0.05$

Cyp2s1 response in these cells. In the early phase of inflammation, PGE2 can attract neutrophils and then serves as pro-inflammatory molecule. ${ }^{25}$ Most importantly, however, PGE2 produced by macrophages affects their function towards an anti-inflammatory tissue reconstituting phenotype. ${ }^{26}$ In line, phagocytosis of fluorescently labeled beads was increased after serum starvation.

\section{Discussion}

Therapeutic angiogenesis is considered as a potential strategy for treating hind-limb ischemia. ${ }^{27}$ However, an efficient induction of mature and functional blood vessels remains still challenging. In previous reports, it was demonstrated that PHD2 expressed in macrophages is able to influence therapeutic angiogenesis after hind-limb ischemia. ${ }^{15}$ In sharp contrast, no similar findings were obtained in our study regarding PHD3 expressed in myeloid cells. Neither
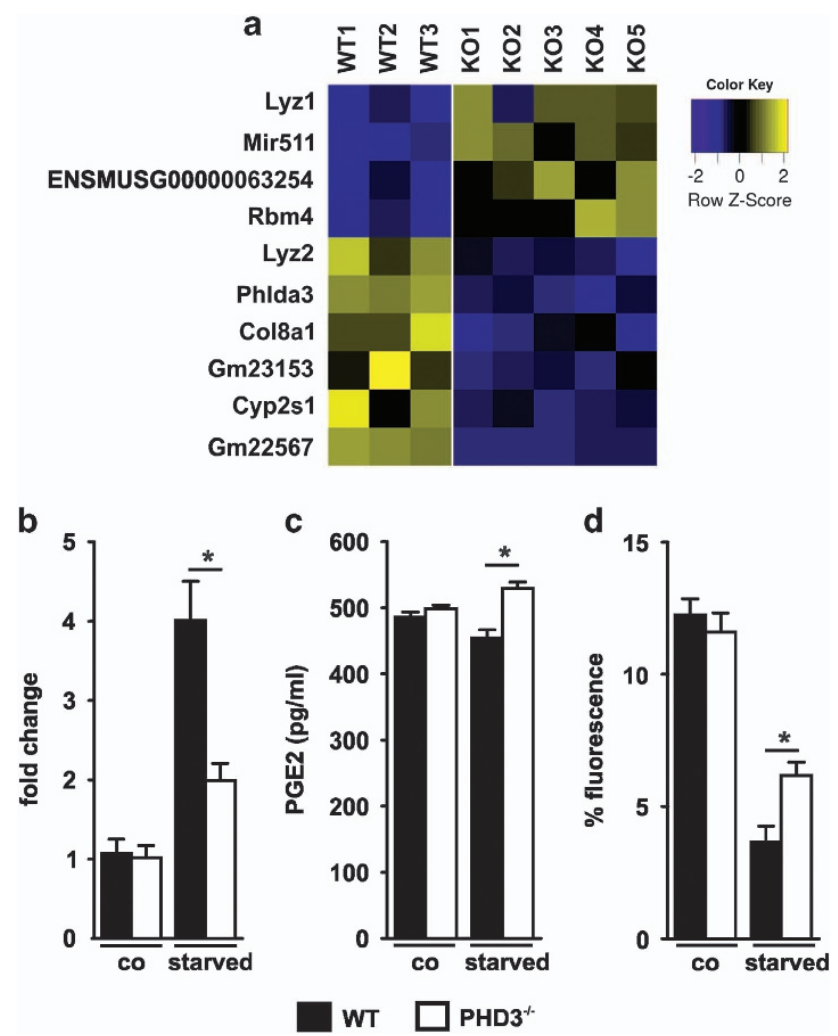

Figure $5 \mathrm{PHD}^{-1-}$ macrophages show a decreased expression of the cytochrome P450 enzyme Cyp2s1, which coincides with a higher PGE2-secretion and an increased phagocytotic capacity in $\mathrm{PHD}^{-1-}$ BMDM upon starvation. (a) F4/80 CD11b-positive macrophages were sorted from gastrocnemius muscles of three wild type (WT) and five $\mathrm{PHD}^{-1-}$ (KO) mice 5 days after hind-limb ischemia surgery and analyzed via RNA-sequencing. In total, four genes were significantly upregulated and six significantly downregulated in $\mathrm{PHD}^{-l-}$ macrophages (log-fold change of at least \pm 1 ). (b) RNA-expression of Cyp2s1 was analyzed in WT and in $\mathrm{PHD}^{-1-}$ bone marrow-derived macrophages (BMDM) upon serum starvation (24 h). (c) Prostaglandin E2 was determined by ELISA in the supernatants of WTand $\mathrm{PHD}^{-1-}$ BMDM (d) Phagocytosis of WT and $\mathrm{PHD}^{-1-}$ BMDM in control conditions or after $24 \mathrm{~h}$ serum starvation. Graphs are representative for at least three independent experiments. Bars show mean \pm S.E.M. with ${ }^{*} P<0.05$

perfusion nor capillary number or average capillary size was altered in the $\mathrm{PHD}^{-1-}$ compared to the WT mice after hindlimb ischemia. This is in line with the previous notion that the three PHD variants in mammalia differ regarding cell typespecific expression but especially regarding function. A compensatory mechanism by PHD3, which limits the HIF response as a consequence of a PHD2 knockout has been described in several cell and animal models. ${ }^{21,28-30}$ This might explain why some phenotypes induced by a PHD2 knockout are more severe in combination with knocking out PHD3. A singular knockout of PHD3 alone, however, seems to reveal the PHD3 isoform-specific functions and might explain, why the phenotype observed in the $\mathrm{PHD}^{-/-}$compared to the WT mice are seen just in the ischemic legs in situ or under simulated ischemic conditions in vitro.

PHD3 has been associated with cell survival decisions and the course of inflammation. ${ }^{31}$ Moreover, PHD3 has been shown to cause apoptosis in neuronal cells and in cancer cells when expressed at high levels. ${ }^{32-35}$ Compared to PHD2, 
PHD3 has been suggested to have non-HIF targets and downstream effectors. ${ }^{36}$ We found in the $\mathrm{PHD}^{-1-}$ mice a dampened inflammatory infiltrate in the ischemic muscle, which contained a lower number of macrophages whereas the number of neutrophils was unaffected. The lower number of macrophages was not due to increased cell death as the number of apoptotic and dead macrophages were not significantly altered in the $\mathrm{PHD}^{-1-}$ ischemic legs. However, the macrophage population switched to a more antiinflammatory type. This was reflected by the increased expression of the M2-marker CD206 as well as the decreased expression of Cyp2s1. Bui et al. identified that human Cyp2s1 is an important enzyme in the metabolism of COXderived prostaglandins at nanomolar concentrations, and the authors suggested that Cyp2s1 may have an important role in modulating the inflammatory process. ${ }^{37,38}$ In macrophages Cyp2s1 is an epoxygenase, which has been described to metabolize PGG2 and PGH2 to $12-\mathrm{HHT}$ and thereby influences PGE2 production. ${ }^{39} \mathrm{PGH} 2$ is synthesized from arachidonic acids by the action of cyclooxygenase- 1 and 2 via the intermediate product PGG2. PGH2 also serves as a substrate for PGE2 synthesis via the PGE2 synthases. ${ }^{40}$ PGE2 is a potent pro- and anti-inflammatory mediator, which in the early inflammatory phase mediates attraction and activation of neutrophils, but has also been described to enhance M2-polarization of macrophages and to interfere with inflammasome activation in sterile inflammation. ${ }^{26,41}$ Cyp2s1 and PGE2 have both been described to affect inflammatory macrophage function like phagocytosis. Downregulation of Cyp2s1 by siRNA led to increased phagocytosis in monocytes. ${ }^{39}$ This is in line with the increased phagocytosis in $\mathrm{PHD}^{-/-}$BMDM found in our study. Most interestingly the effects of altered Cyp2s1 expression, PGE2 production and altered phagocytosis were seen in the BMDM under starved conditions only. In contrast to PHD1 and PHD2, PHD3 is highly inducible in hypoxia. Aside from this, PHD3 is also upregulated in other (patho)physiological conditions like vascular tissue injury, ${ }^{42}$ ageing ${ }^{43}$ and especially nerve growth factor removal. $^{32-44}$ In neural cells, PHD3 is required and is even sufficient to induce apoptosis after growth factor removal. Serum starvation as employed in our study might mimic at least in part the in vivo situation of the macrophages in the tissue after hind-limb ischemia. Although we can formally not delineate, whether the role of PHD3 for Cyp2s1 expression in vitro in consequence of serum starvation is mainly due to the fact, that these conditions foster PHD3 expression or whether additional factors are induced, these experiments demonstrate that studies, which rely on BMDM under standard cell culture conditions only, might not be able to reveal PHD3related functions fully. Some features might just uncover in the in vivo situation mediated by the interplay with other immune cells or special tissue conditions like nutrient depletion. This applies to the identified anti-inflammatory Cyp2s1 expression profile and phagocytosis observed in the $\mathrm{PHD}^{-/-}$macrophages. Overall, the anti-inflammatory phenotype in the ischemic leg in the $\mathrm{PHD}^{-/-}$mice was associated with a decrease in fibrosis.

Small molecule inhibitors of the PHD enzymes are explored regarding their use in the context of ischemic diseases. ${ }^{16}$ The inhibitors applied so far, are not selective regarding PHD isoforms. However, it is worthwhile to know, which isoform is mediating tissue protective function and which cell types are involved. According to our data, inhibition of PHD3 activity in macrophages might not affect therapeutic angiogenesis it might however indeed affect the non-sterile inflammation.

\section{Materials and Methods}

Mice. Generation of LysM Cre+l- $x$ PHD3fl/fl $\left(\mathrm{PHD}^{-1-}\right)$ mice and LysM $\mathrm{Cre}^{-I-} \times \mathrm{PHD}^{\text {fl/fl }}(\mathrm{WT})$ littermate control mice were described previously. ${ }^{18}$ Animals were maintained on a $\mathrm{C} 57 \mathrm{BL} / 6$ background.

Hind-limb ischemia. All animal experiments were performed according to the German animal protection law and approved by the Niedersächsische Landesamt für Verbraucherschutz und Lebensmittelsicherheit in Oldenburg (animal experimentation numbers 33.942502-04-16/2139 and 33.9-42502-04-11/0413). Unilateral hind-limb ischemia was performed as described by Limbourg et al. ${ }^{12}$ In brief, male mice at the age of 8-12 weeks were anaesthetized by intraperitoneal injection of $15 \mathrm{mg} / \mathrm{kg}$ body weight xylazin, $75 \mathrm{mg} / \mathrm{kg}$ body weight ketamine in $0.9 \%$ sodium chloride and placed on a $37^{\circ} \mathrm{C}$ heating pad. Through two longitudinal incisions above the knee and at the groin the $\mathrm{A}$. femoralis was exposed and occluded proximal and distal to the A. profunda femoris with silk sutures using triple surgical knots. The part of the artery between the knots was removed without damaging the femoral vein and nerve and the skin was sutured. This surgery treatment enables the induction of severe ischemia in the M. gastrocnemius (GM) and analysis of the resulting angiogenesis, but precludes arteriogenesis development by pre-existing collateral arterioles in the skeletal muscles of the thigh.

Laser doppler perfusion imaging. Blood perfusion was measured using PeriScan PIM3 laser Doppler (Perimed Instruments, Cologne, Germany). For the scanning procedure mice were anaesthetized by intraperitoneal injection of $15 \mathrm{mg} /$ $\mathrm{kg}$ body weight xylazin, $75 \mathrm{mg} / \mathrm{kg}$ body weight ketamine in $0.9 \%$ sodium chloride and placed on a $37^{\circ} \mathrm{C}$ heating pad. Perfusion rate was analyzed using the PIMsoft 1.3 software (Perimed Instruments, Cologne, Germany) by comparing the perfusion units, calculated from the heat map of defined regions of interest of the ligated versus non-ligated leg.

Analysis of capillary density and size. Cryosections were fixed for $20 \mathrm{~min}$ in a $1: 1$ acetone/methanol solution at $-20^{\circ} \mathrm{C}$. Incubation in blocking solution ( $10 \%$ normal goat serum, $0.1 \%$ Triton $X$ in PBS) was followed by staining of the sections with 1:200 Alexa488-coupled isolectin GS-IB4 (I21411, Invitrogen, Darmstadt, Germany) and 1:100 anti-vinculin antibodies (V9264, Sigma, St. Louis, MO, USA). After extensive washing with PBS, slides were incubated with 1:200 TRcoupled anti-mouse antibodies (Sc-2781, Santa Cruz Biotechnology, Santa Cruz, CA, USA). Sections were washed in PBS and stained using 1:500 Hoechst (Thermo Scientific, Waltham, MA, USA) for 10 min. Subsequently, samples were embedded in Mowiol (3\% glycerin, 1.33\% Mowiol, 0.133 Tris pH 8.5, 0.1\% DABCO). Stained sections were imaged by fluorescence microscopy (Zeiss Observer D1 microscope, Carl Zeiss, Göttingen, Germany). 15-20 images were taken of different areas of the muscle. The amount and average size of capillaries were then analyzed using ImageJ (U.S. National Institutes of Health, Bethesda, MD, USA).

Picro-sirius red staining. Cryosections of GM were placed in xylol and subsequently in $99-60 \%$ ethanol. Slides were incubated in $0.1 \%$ Picro-sirius red staining solution (Sigma) for $60 \mathrm{~min}$. After washing with 1\% acetic acid, sections were incubated in ethanol and xylol and embedded in Roti-Histo-kitt (Roth). The whole section was imaged (Zeiss Observer D1 microscopy) and analyzed for Picrosirius red positive areas using ImageJ.

Isolation and differentiation of bone marrow-derived macrophages. Bone marrow-derived macrophages were isolated and differentiated as described previously. ${ }^{18}$ Resulting cell suspension was cultivated for $48 \mathrm{~h}$. Purity of the adherent macrophages were analyzed by F4/80 and CD11b staining and FACS analysis.

Hypoxic incubation. For culturing cells in defined hypoxic conditions $\left(1 \% \mathrm{O}_{2}\right)$ an in vivo Hypoxia workstation (Ruskinn Technologies, Bridgend, South Wales, UK) was used. 
Single cell migration. Migration capacity was determined using a single-cell migration approach, which studies random migration as described before. ${ }^{21}$

Macrophage viability. To quantify viability of the FACS analyzed macrophages in the digested GM, F4/80 CD11b double-positive cells were stained with Pacific blue coupled Annexin V (1:50, Biolegend) and FITC coupled Zombie Green (1:1000, Biolegend, San Diego, CA, USA). Annexin V-/Zombie Green low, Annexin $\mathrm{V}+$ /Zombie Green low, Annexin V+/Zombie green high and Annexin V-/Zombie Green high $F 4 / 80+C D 11 b+$ cells were regarded to be viable, early apoptotic, late apoptotic and dead, respectively.

FACS analysis. GM were removed from the legs of mice, cut into pieces, which were suspended in digestion buffer $(450 \mathrm{U} / \mathrm{ml}$ collagenase IV, $1.2 \mathrm{U} / \mathrm{ml}$ dispase II in PBS) and incubated for $45 \mathrm{~min}$ at $37^{\circ} \mathrm{C}$ with vigorous pipetting every $15 \mathrm{~min}$. After stopping the digestion with DMEM containing $15 \% \mathrm{FCS}, 1 \% \mathrm{p} / \mathrm{s}$ samples were filtered using a $40 \mu \mathrm{m}$ mesh, centrifuged, washed in PBS and counted. Overall, $300000-$ 500000 cells per sample were used for further analysis. Non-specific Fc binding sites were blocked with Mouse BD Fc Block (Biolegend). For analysis of cell subpopulations fluorescently coupled antibodies targeting the following surface markers were added, neutrophils Ly-6G-FITC, CD11b-PE/Cy7; macrophages F4/80-FITC, CD11b-PE/Cy7; M1 polarization F4/80-FITC, CD11b-PE/Cy7, CD86-PE; M2-polarization F4/80-FITC, CD11b-PE/Cy7, CD206-APC. All antibodies and their respective isotype controls were purchased from Biolegend. Stained cells were analyzed using the FACS Canto II flow cytometer (BD Biosciences, Heidelberg, Germany). A compensation was calculated for all staining protocols.

RNA isolation and qRT-PCR. RNA was isolated using TRIzol (Thermo Fisher Scientific, Waltham, MA, USA). RNA was transcribed into cDNA using the First strand CDNA synthesis kit (Thermo Fisher Scientific). qRT-PCR was performed using the SensiMix SYBR Low-ROX kit according to the manufacturer's instructions. The following primers were applied: Cyp2s1: $5^{\prime}$-TGCTCCTGCTGAGATACCCT-3' and 5'-GAAGCAAGTGGTCCTCGTGA-3', S12: 5'-GAAGCTGCCAAGGCCTTAGA $-3^{\prime}$ and $5^{\prime}$-AACTGCAACCAACCACCTTC-3', PHD3: 5'-GGCCGCTGTATCACCTG TAT- $3^{\prime}$ and $5^{\prime}$-TTCTGCCCTTTCTTCAGCAT-3'

Transcriptome analysis. GM lysates were washed and then stained with the fluorescently labeled F4/80-PE and CD11b APC antibodies. Doublets were discriminated using SSC-A versus SSC-W gating. The cell population positive for both markers were selected and sorted via a FACS Aria III flow cytometer (BD Biosciences, Heidelberg, Germany) into $1 \mathrm{ml}$ TRlzol. RNA was isolated using chloroform/isopropanol. RNA was prepared for sequencing using the TruSeq total RNA sample preparation kit (lllumina, San Diego, CA, USA). This includes rRNA depletion, fragmentation of RNA and synthesis of cDNA. Modified cDNA was amplified in a PCR reaction and sequenced using the lllumina technique. The results of the total RNAsequencing were analyzed using DeSeq2 software (Illumina, San Diego, CA, USA). Only genes that showed a significant log2 fold change of more than 1 or less than -1 were considered as candidate RNAs. Statistical significance was determined using the Benjamin-Hochberg correction. Adjusted $P$-values $<0.05$ were considered statistically significant.

Prostaglandin E2 ELISA. Prostaglandin E2 (PGE2) levels in cell culture supernatants were measured using a chemiluminescent ELISA kit according to the manufacturer's recommendation (Abcam, Cambridge, UK).

Phagocytosis. Phagocytotic capacity was analyzed using fluorescently labeled beads as described previously. ${ }^{21}$

Statistics. Statistical significance between the two different genotypes was calculated by using an unpaired two-tailed student's $t$-test. A significant difference between two means was defined by a $P$-value $<0.05$.

\section{Conflict of Interest}

The authors declare no conflict of interest.

Acknowledgements. $A B$ and $A G$ are fellows of the IRTG 1816 funded by the Deutsche Forschungsgemeinschaft.

\section{Publisher's Note}

Springer Nature remains neutral with regard to jurisdictional claims in published maps and institutional affiliations.

1. Kono $\mathrm{H}$, Onda A, Yanagida T. Molecular determinants of sterile inflammation. Curr Opin Immunol 2014; 26: 147-156.

2. Arslan F, de Kleijn DP, Pasterkamp G. Innate immune signaling in cardiac ischemia. Nat Rev Cardiol 2011; 8: 292-300.

3. Chambers SE, O'Neill CL, O'Doherty TM, Medina RJ, Stitt AW. The role of immune-related myeloid cells in angiogenesis. Immunobiology 2013; 218: 1370-1375.

4. Paoni NF, Peale F, Wang F, Errett-Baroncini C, Steinmetz H, Toy K et al. Time course of skeletal muscle repair and gene expression following acute hind limb ischemia in mice. Physiol Genomics 2002; 11: 263-272.

5. Taylor CT, Doherty G, Fallon PG, Cummins EP. Hypoxia-dependent regulation of inflammatory pathways in immune cells. J Clin Invest 2016; 126: 3716-3724.

6. Greer SN, Metcalf JL, Wang Y, Ohh M. The updated biology of hypoxia-inducible factor. Embo J 2012; 31: 2448-2460.

7. Bishop T, Ratcliffe PJ. Signaling hypoxia by hypoxia-inducible factor protein hydroxylases: a historical overview and future perspectives. Hypoxia 2014; 2: 197-213.

8. Kaelin WG Jr., Ratcliffe PJ. Oxygen sensing by metazoans: the central role of the HIF hydroxylase pathway. Mol Cell 2008; 30: 393-402.

9. Schödel J, Mole DR, Ratcliffe PJ. Pan-genomic binding of hypoxia-inducible transcription factors. Biol Chem 2013; 394: 507-517.

10. Eltzschig HK, Bratton DL, Colgan SP. Targeting hypoxia signalling for the treatment of ischaemic and inflammatory diseases. Nat Rev Drug Discov 2014; 13: 852-869.

11. Selvaraju V, Parinandi NL, Adluri RS, Goldman JW, Hussain N, Sanchez JA et al. Molecular mechanisms of action and therapeutic uses of pharmacological inhibitors of HIF-prolyl 4-hydroxylases for treatment of ischemic diseases. Antioxid Redox Signal 2014; 20 : 2631-2665.

12. Limbourg A, Korff T, Napp LC, Schaper W, Drexler H, Limbourg FP. Evaluation of postnatal arteriogenesis and angiogenesis in a mouse model of hind-limb ischemia. Nat Protoc 2009; 4: $1737-1746$

13. Loinard C, Ginouves A, Vilar J, Cochain C, Zouggari Y, Recalde A et al. Inhibition of prolyl hydroxylase domain proteins promotes therapeutic revascularization. Circulation 2009; 120 : 50-59.

14. Paik KJ, Maan ZN, Zielins ER, Duscher D, Whittam AJ, Morrison SD et al. Short hairpin RNA silencing of PHD-2 improves neovascularization and functional outcomes in diabetic wounds and ischemic limbs. PLOS ONE 2016; 11: e0150927.

15. Takeda Y, Costa S, Delamarre E, Roncal C, Leite de Oliveira R, Squadrito ML et al. Macrophage skewing by Phd2 haplodeficiency prevents ischaemia by inducing arteriogenesis. Nature 2011; 479: 122-126.

16. Katschinski DM. In vivo functions of the prolyl-4-hydroxylase domain oxygen sensors: direct route to the treatment of anaemia and the protection of ischaemic tissues. Acta Physiol 2009; 195: 407-414.

17. Harris AJ, Thompson AR, Whyte MK, Walmsley SR. HIF-mediated innate immune responses: cell signaling and therapeutic implications. Hypoxia 2014; 2: 47-58.

18. Swain L, Wottawa M, Hillemann A, Beneke A, Odagiri H, Terada K et al. Prolyl-4-hydroxylase domain $3(\mathrm{PHD} 3)$ is a critical terminator for cell survival of macrophages under stress conditions. J Leukoc Biol 2014; 96: 365-375.

19. Walmsley SR, Chilvers ER, Thompson AA, Vaughan K, Marriott HM, Parker LC et al. Prolyl hydroxylase 3 (PHD3) is essential for hypoxic regulation of neutrophilic inflammation in humans and mice. J Clin Invest 2011; 121: 1053-1063.

20. Kiss J, Mollenhauer M, Walmsley SR, Kirchberg J, Radhakrishnan P, Niemietz T et al. Loss of the oxygen sensor PHD3 enhances the innate immune response to abdominal sepsis. J Immunol 2012; 189: 1955-1965.

21. Guentsch A, Beneke A, Swain L, Farhat K, Nagarajan S, Wielockx B et al. PHD2 is a regulator for glycolytic reprogramming in macrophages. Mol Cell Biol 2017; 37: e00236-16

22. Verdeguer F, Aouadi M. Macrophage heterogeneity and energy metabolism. Exp Cell Res 2017: 17: 30162-30163

23. Saarikoski ST, Rivera SP, Hankinson O, Husgafvel-Pursiainen K. CYP2S1: a short review. Toxicol Appl Pharmacol 2005; 207: 62-69.

24. Spector AA. Arachidonic acid cytochrome P450 epoxygenase pathway. J Lipid Res 2009; 50: S52-S56.

25. Yu Y, Chadee K. Prostaglandin E2 stimulates IL-8 gene expression in human colonic epithelial cells by a posttranscriptional mechanism. J Immunol 1998; 161: 3746-3752.

26. Strassmann G, Patil-Koota V, Finkelman F, Fong M, Kambayashi T. Evidence for the involvement of interleukin 10 in the differential deactivation of murine peritoneal macrophages by prostaglandin E2. J Exp Med 1994; 180: 2365-2370.

27. Cooke JP, Losordo DW. Modulating the vascular response to limb ischemia: angiogenic and cell therapies. Circ Res 2015; 116: 1561-1578.

28. Minamishima YA, Moslehi J, Padera RF, Bronson RT, Liao R, Kaelin WG Jr. A feedback loop involving the Phd3 prolyl hydroxylase tunes the mammalian hypoxic response in vivo. $\mathrm{Mol}$ Cell Biol 2009; 29: 5729-5741. 
29. Appelhoff RJ, Tian YM, Raval RR, Turley H, Harris AL, Pugh CW et al. Differential function of the prolyl hydroxylases PHD1, PHD2, and PHD3 in the regulation of hypoxia-inducible factor. J Biol Chem 2004; 279: 38458-38465.

30. Urrutia AA, Afzal A, Nelson J, Davidoff O, Gross KW, Haase VH. Prolyl-4-hydroxylase 2 and 3 coregulate murine erythropoietin in brain pericytes. Blood 2016; 128: 2550-2560.

31. Place TL, Domann FE. Prolyl-hydroxylase 3: evolving roles for an ancient signaling protein. Hypoxia 2013; 2013: 13-17.

32. Lee S, Nakamura E, Yang H, Wei W, Linggi MS, Sajan MP et al. Neuronal apoptosis linked to EgIN3 prolyl hydroxylase and familial pheochromocytoma genes: developmental culling and cancer. Cancer Cell 2005; 8: 155-167.

33. Lipscomb EA, Sarmiere PD, Crowder RJ, Freeman RS. Expression of the SM-20 gene promotes death in nerve growth factor-dependent sympathetic neurons. J Neurochem 1999; 73: 429-432.

34. Rantanen K, Pursiheimo J, Hogel H, Himanen V, Metzen E, Jaakkola PM. Prolyl hydroxylase PHD3 activates oxygen-dependent protein aggregation. Mol Biol Cell 2008; 19: 2231-2240.

35. Schlisio S, Kenchappa RS, Vredeveld LC, George RE, Stewart R, Greulich H et al. The kinesin KIF1Bbeta acts downstream from EgIN3 to induce apoptosis and is a potential 1p36 tumor suppressor. Genes Dev 2008; 22: 884-893.

36. Jaakkola PM, Rantanen K. The regulation, localization, and functions of oxygen-sensing prolyl hydroxylase PHD3. Biol Chem 2013; 394: 449-457.

37. Bui P, Imaizumi S, Beedanagari SR, Reddy ST, Hankinson O. Human CYP2S1 metabolizes cyclooxygenase- and lipoxygenase-derived eicosanoids. Drug Metab Dispos 2011; 39: 180-190.

38. Bui PH, Hankinson O. Functional characterization of human cytochrome P450 2S1 using a synthetic gene-expressed protein in Escherichia coli. Mol Pharmacol 2009; 76: 1031-1043.

39. Fromel T, Kohlstedt K, Popp R, Yin X, Awwad K, Barbosa-Sicard E et al. Cytochrome P4502S1: a novel monocyte/macrophage fatty acid epoxygenase in human atherosclerotic plaques. Basic Res Cardiol 2013; 108: 319
40. Kalinski P. Regulation of immune responses by prostaglandin E2. J Immunol 2012; 188: 21-28.

41. Sokolowska M, Chen LY, Liu Y, Martinez-Anton A, Qi HY, Logun C et al. Prostaglandin E2 inhibits NLRP3 inflammasome activation through EP4 receptor and intracellular cyclic AMP in human macrophages. J Immunol 2015; 194: 5472-5487.

42. Wax SD, Tsao L, Lieb ME, Fallon JT, Taubman MB. SM-20 is a novel 40-kd protein whose expression in the arterial wall is restricted to smooth muscle. Lab Invest 1996; 74: 797-808.

43. Rohrbach S, Simm A, Pregla R, Franke C, Katschinski DM. Age-dependent increase of prolyl-4-hydroxylase domain (PHD) 3 expression in human and mouse heart. Biogerontology 2005; 6: 165-171.

44. Straub JA, Lipscomb EA, Yoshida ES, Freeman RS. Induction of SM-20 in PC12 cells leads to increased cytochrome $c$ levels, accumulation of cytochrome $c$ in the cytosol, and caspase-dependent cell death. J Neurochem 2003; 85: 318-328.

(c) Cell Death and Disease is an open-access journal published by Nature Publishing Group. This work is

licensed under a Creative Commons Attribution 4.0 International License. The images or other third party material in this article are included in the article's Creative Commons license, unless indicated otherwise in the credit line; if the material is not included under the Creative Commons license, users will need to obtain permission from the license holder to reproduce the material. To view a copy of this license, visit http://creativecommons.org/licenses/by/4.0/

(C) The Author(s) 2017 